\title{
Chemiluminescent Determination of Peroxidase with Purpurogallin Carboxylic Acid
}

\author{
Tadashi Segawa, Takahiro Ooizumi, Taichi Matsubara, Tamio Kamidate and Hiroto Watanabe \\ Faculty of Engineering, Hokkaido University, Sapporo 060, Japan
}

\begin{abstract}
Purpurogallin carboxylic acid (PurA) was proposed as a highly sensitive chemiluminescent $(\mathrm{CL})$ reagent for the detection of peroxidase. The blank signal of PurA was lower than that of its mother moiety, purpurogallin, thus resulting in a decrease in the detection limit of peroxidase. The calibration curves of horseradish peroxidase and Arthromyces ramosus peroxidase with PurA were linear over the ranges from a detection limit of $20 \mathrm{fmol}$ to $5.0 \mathrm{pmol}$ and $10 \mathrm{fmol}$ to $5.0 \mathrm{pmol}$, respectively. PurA CL was less subject to interference from diverse albumin than was luminol CL.
\end{abstract}

Keywords Chemiluminescence, polyphenol, peroxidase

Peroxidase (EC 1.11.1.7; POD) is widely used for a non-radioactive label in an enzyme immunoassay and a DNA dot-hybridization assay. ${ }^{1-3}$ Of PODs, horseradish POD (HRP) is accepted to be a versatile label for the assays, because HRP is easily available. HRP has been determined by both a colorimetric method and a fluorometric method. ${ }^{1,2}$ Recently, a chemiluminescent (CL) method for HRP detection has been noted in view of its high sensitivity and wide dynamic range. For this purpose, luminol ${ }^{4,5}$ and polyphenolic compounds, such as pyrogallol (PG) $)^{6-8}$ and purpurogallin (Pur) ${ }^{9}$, are used. In luminol $C L$, an excellent enhancer, such as $p$ iodophenol, was found. ${ }^{10,11}$ Subsequently, many assay kits for biological substances with the enhanced-luminol CL have been developed. ${ }^{12}$

These days, ultra-weak light emission can easily be detected by a photomultiplier tube (PMT). Hence, in a $\mathrm{CL}$ analysis using a PMT, a strongly emissive $\mathrm{CL}$ reagent is not necessary if the CL signal of a sample solution and that of a blank solution are clearly distinctive. We should thus pay attention to the lower blank CL for developing a sensitive $C L$ method.

In HRP-catalyzed luminol CL, the blank CL signals are significant, because of impurities in luminol. Kricka et al., attempted the recrystallization of luminol for reducing the blank CL. ${ }^{13}$ However, their effort was unsuccessful, since the blank CL signal still remained considerable. On the other hand, no reports have been found in an attempt to decrease the blank CL in the HRP-catalyzed polyphenol CL.

During the course of our studies on POD-catalyzed polyphenol CL we have found that carboxyl derivatives of PG and Pur were a good choice for decreasing the blank CL. Hence, in the present study we tested the carboxyl derivatives of Pur for the sensitive CL determination of PODs.

\section{Experimental}

\section{Apparatus}

All of the CL measurements were made with a CL detector (TD-3A; Tohoku Denshi Sangyo Co., Ltd.) equipped with an automatic injector (Model 500, Nichiryo Co., Ltd.). The absorption spectra and the absorbance were recorded on a spectrometer (U-2000; Hitachi Co., Ltd.)

\section{Reagents}

Figure 1 lists the polyphenol compounds tested. 7 Carboxy-2,3,4,6-tetrahydroxy-5 $H$-benzocyclohepten-5one (PurA) was synthesized according to a procedure of Crow et al..$^{14}$ Aqueous solutions of PG (15.5 g in 160 $\mathrm{cm}^{3}$ ) and potassium iodate ( $27.1 \mathrm{~g}$ in $330 \mathrm{~cm}^{3}$ ) were added simultaneously with stirring and ice-cooling to a $200 \mathrm{~cm}^{3}$ portion of an aqueous solution containing $21.0 \mathrm{~g}$ of gallic acid (GA) and $11.5 \mathrm{~g}$ of sodium hydrogen carbonate within $23 \mathrm{~min}$. After stirring for an additional $15 \mathrm{~min}$ the mixture was acidified with $12 \mathrm{M}\left(1 \mathrm{M}=1 \mathrm{~mol} \mathrm{dm}^{-3}\right)$ hydrochloric acid $\left(10 \mathrm{~cm}^{3}\right)$, set overnight and filtered. The filtrate was then extracted with diethylether $\left(1 \mathrm{dm}^{3}\right)$. The ethereal solution was dryed, and an orange powder of PurA was obtained.

All other reagents were used as purchased without any further purification. HRP-Type VI was obtained from Sigma Chemical Co. Arthromyces ramosus POD (ARP) was obtained from Suntory Ltd. All of the aqueous solutions were prepared with water purified using a Millipore Milli-Q Labo system.

An $\mathrm{H}_{2} \mathrm{O}_{2}$ solution was prepared by diluting a $31 \%$ $\mathrm{H}_{2} \mathrm{O}_{2}$ solution with a $0.10 \mathrm{M}$ 2-morpholinoethanesulfonic acid (MES) buffer solution adjusted to $\mathrm{pH}$ 7.0. 
<smiles>Oc1cccc(O)c1O</smiles>

Pyrogallol(PG)<smiles>O=c1c(O)cccc2cc(O)c(O)c(O)c12</smiles>

Purpurogallin(Pur)<smiles>O=C(O)c1cc(O)c(O)c(O)c1</smiles>

Gallic acid(GA)<smiles>O=C(O)c1cc(O)c(=O)c2c(O)c(O)cc(O)c2c1</smiles>
Purpurogallin carboxylic acid (Pur A)

Fig. 1 Polyphenol compounds tested.

\section{Procedure}

A $0.10 \mathrm{~cm}^{3}$ portion of a POD solution and a $0.40 \mathrm{~cm}^{3}$ portion of a $C L$ reagent solution were introduced into a glass cuvette $(22 \mathrm{~mm}$ i.d. $\times 20 \mathrm{~mm})$, which was subsequently placed in the holder of the CL counter. A $0.50 \mathrm{~cm}^{3}$ portion of the $\mathrm{H}_{2} \mathrm{O}_{2}$ solution was then added into the cuvette using the injector. The $\mathrm{CL}$ reaction was thus initiated, and the CL emission was monitored as a function of time with a PMT. The resultant photocurrent was converted to a voltage, whose value was displayed on a chart recorder. The CL intensity was defined as the voltage at which the $\mathrm{CL}$ emission was maximum.

All of the reagent concentrations in the figure captions are final values.

\section{Results and Discussion}

\section{CL response of $P G$ and Pur}

Typical CL response curves of $P G$ and Pur were measured by using a $0.30 \mathrm{pmol}$ HRP solution. The results are shown in Fig. 2. When PG was used, light emission occurred several seconds after the start of the $\mathrm{CL}$ reaction (Fig. 2, curve 1). The intensities of the light emission gradually increased, reaching a maximum value, and then decreased. On the other hand, when Pur was used, CL appeared instantaneously after the initiation of the reaction (Fig. 2, curve 2). The CL emission reached a maximum value after about $1 \mathrm{~min}$, and then decreased. The blank CL response curves of PG and Pur are also shown in Fig. 1, curves 3 and 4. Pur gave a higher CL intensity and blank CL than did PG.

Recently, the luminol CL method has been modified by the use of POD from different origins such as fungus ${ }^{15}$, for improving the sensitivity. However, the use of POD is only limited to HRP as a catalyst in the polyphenol CL reaction.

We then examined the polyphenol CL by using
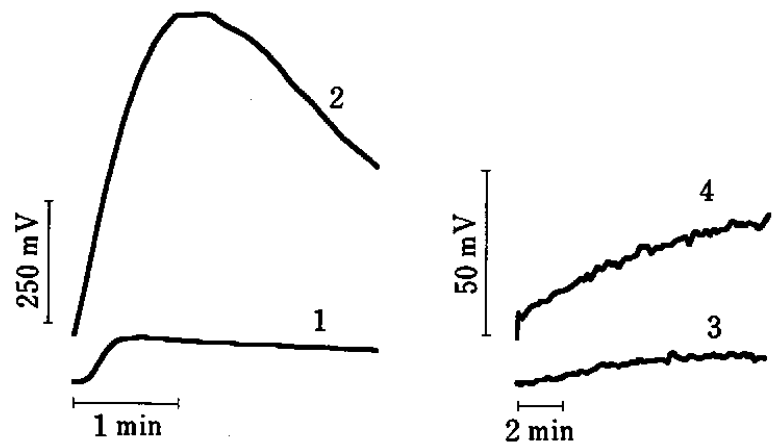

Fig. 2 Typical CL response curves of $P G$ and Pur. $\mathrm{HRP}=0.30 \mathrm{pmol} ;\left[\mathrm{H}_{2} \mathrm{O}_{2}\right]=3.0 \times 10^{-2} \mathrm{M} ; \mathrm{pH} 7.0$, curves 1 and 3 , $P G=1.2 \times 10^{-2} \mathrm{M}$; curves 2 and $4, P u r=6.0 \times 10^{-4} \mathrm{M}$. Curves 3 and 4 are the blank $C L$ in the absence of HRP.

Table $1 \mathrm{CL}$ intensity with several polyphenols

\begin{tabular}{lccc}
\hline & Blank $/ \mathrm{mV}$ & HRP $/ \mathrm{mV}$ & ARP $/ \mathrm{mV}$ \\
\hline PG & 25 & 78 & 60 \\
Pur & 45 & 131 & 180 \\
GA & 3 & 3 & 65 \\
Pur A & 19 & 243 & 250 \\
\hline
\end{tabular}

$\mathrm{POD}=0.10 \mathrm{pmol} ;[\mathrm{GA}]=1.2 \times 10^{-2} \mathrm{M} ;\left[\mathrm{H}_{2} \mathrm{O}_{2}\right]=3.0 \times 10^{-2} \mathrm{M}$; $[\mathrm{Pur}]=6.0 \times 10^{-4} \mathrm{M} ;[\mathrm{PG}]=1.0 \times 10^{-2} \mathrm{M} ;[\mathrm{Pur} \mathrm{A}]=5.0 \times 10^{-4} \mathrm{M}$.

0.10 pmol POD solutions of HRP and ARP. In addition, the CL reaction was made by using water in place of the POD solution. Table 1 summarized the CL intensities of PG and Pur for the blank and PODs (line 1 and 2). ARP gave results similar to those in HRP.

The blank CL intensity of Pur was greater than that of PG. Since the detection limit of POD is commonly defined as moles of POD yielding a CL intensity equal to two- or three-times the blank CL intenisty, the decrease in the blank CL emission provides a good means for increasing the sensitivity for POD. Then, the mechanism of PG-CL and Pur-CL was investigated.

\section{CL mechanism of $P G$ and Pur}

The speculated CL mechanisms of PG and Pur are shown in Fig. 3.6,16,17 Polyphenol CL is believed to emit light from singlet oxygen, which is produced during the oxidation of the polyphenolic compound. In PG-CL, PG is oxidized with POD and $\mathrm{H}_{2} \mathrm{O}_{2}$ to convert into Pur radical (reaction (1)). The Pur radical is then oxidized by $\mathrm{H}_{2} \mathrm{O}_{2}$ without $\mathrm{POD}$; then, singlet oxygen is produced (reaction (2)). Light emission occurs when the singlet oxygen returns to its ground triplet state (reaction (3)). Meanwhile, in the Pur-CL, Pur is oxidized with POD and $\mathrm{H}_{2} \mathrm{O}_{2}$ to form the Pur radical (reaction ( $\left(1^{\prime}\right)$ ). $\quad \mathrm{CL}$ is emitted in a manner similar to the PG-CL (reactions (2) and (3)). In the presence of a trace amount of POD, the rate-limiting step could be the oxidation of PG with HRP 


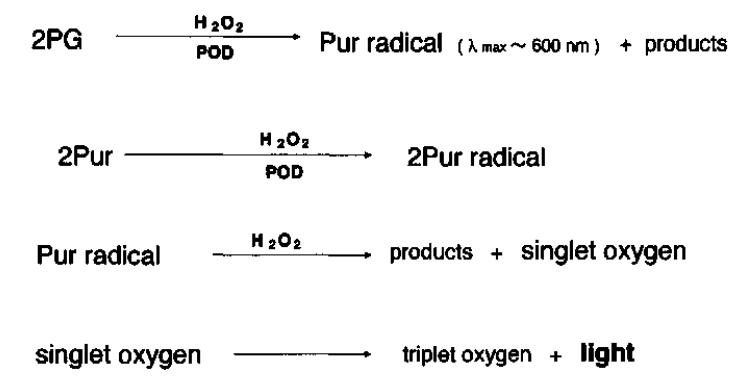

Fig. 3 Reaction scheme of PG and Pur. PG: Eqs. (1), (2) and (3), Pur: Eqs. (1'), (2) and (3).

and $\mathrm{H}_{2} \mathrm{O}_{2}$ in the PG-CL. On the other hand, the ratelimiting step of Pur-CL could be the oxidation of the Pur radical with $\mathrm{H}_{2} \mathrm{O}_{2}$. This asssumption is supported by the fact that PG-CL was not observed from the initiation of the reaction (Fig. 2 curve 1). This fact can be explained in terms of the slow production of the Pur radical in PG-CL (reaction (1)), compared with Pur-CL (reaction $\left(1^{\prime}\right)$ ).

Since blank CL emission is accompanied by the noncatalytic oxidation with $\mathrm{H}_{2} \mathrm{O}_{2}$, a CL reagent which is hardly subject to oxidation gives rise to a low blank CL. In general, the oxidation potential of a phenolic compound increases due to the introduction of an electron-withdrawing substituent, such as a carboxyl group $;^{18}$ thus, phenolic compounds posessing a carboxyl group could be hard to oxidize. For example, the oxidation potential of GA is at least $160 \mathrm{mV}$ higher than that of $\mathrm{PG}$ over the $\mathrm{pH}$ range from 3 to $10 .{ }^{19}$.

\section{$C L$ responses of carboxy derivatives of $P G$ and Pur}

The effects of the carboxyl group on the CL intensity and the blank $C L$ intensity were investigated by using PG and GA. Table 1 also lists the $\mathrm{CL}$ intensity of GA (line 3). The blank CL intensity of GA was much less than that of PG. The increase in the oxidation potential may be reflected on the decrease in the blank CL intensity. In POD-catalyzed CL, GA did not chemiluminesce with $0.10 \mathrm{pmol}$ HRP. On the other hand, the ARPcatalyzed CL of GA and $P G$ gave similar $C L$ intensities. The results of ARP indicate that the carboxyl substituent is effective for a depression of the blank CL.

From these results we should choose a CL reagent which satisfies the dual properties of emitting strong $C L$ and of giving a low blank CL. In this respect, the carboxyl derivative of Pur (PurA) has a potentially higher sensitivity than does Pur. Thus, PurA was synthesized, and the CL intensities of a blank and a $0.10 \mathrm{pmol}$ POD solution were observed. The results are also listed in Table 1 (line 4). The CL intensity of PurA was greater than that of Pur, whereas the blank CL intensity of PurA was less than that of Pur, even PG.

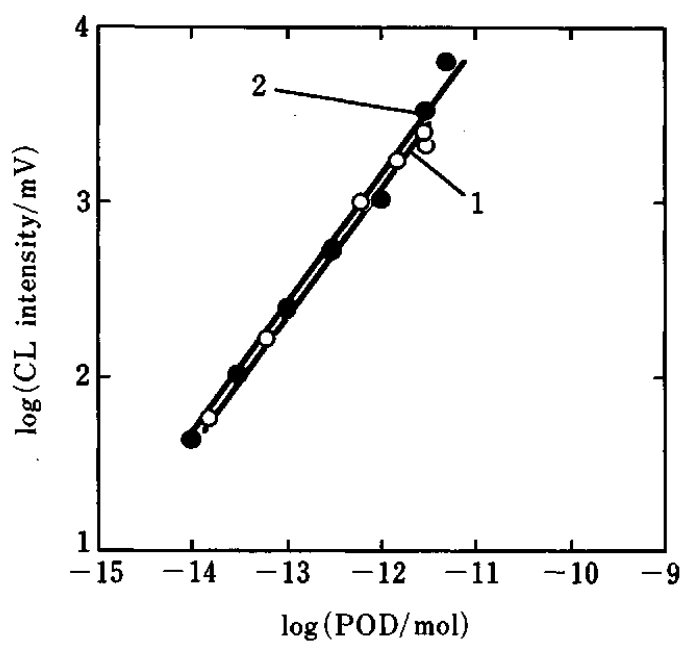

Fig. 4 Calibration curves for POD with PurA. Curve 1, HRP; curve 2, ARP; [PurA] $=5.0 \times 10^{-4} \mathrm{M} ;\left[\mathrm{H}_{2} \mathrm{O}_{2}\right]=3.0 \times$ $10^{-2} \mathrm{M} ; \mathrm{pH} 7.0$.

\section{$C L$ detection of $A R P$ and HRP with PurA}

The optimum conditions for the determination of ARP with PurA were investigated. The effect of the $\mathrm{pH}$ on the $\mathrm{CL}$ intensity was examined in the $\mathrm{pH}$ range from 6.0 to 8.0. The $\mathrm{CL}$ intensity was maximum at $\mathrm{pH} 7.5$. However, the blank CL intensity increased along with an increase in the $\mathrm{pH}$, and greatly increased above $\mathrm{pH}$ 7.0. A buffer of $\mathrm{pH} 7.0$ was therefore chosen for the following studies.

The variation in the CL intensity with the PurA concentration was investigated over a PurA concentration range from $1.0 \times 10^{-4} \mathrm{M}$ to $1.0 \times 10^{-3} \mathrm{M}$. The CL intensity increased along with an increase in the PurA concentration, and reached almost a constant value above $5.0 \times 10^{-4} \mathrm{M}$ PurA. Meanwhile, the blank CL intensity increased together with increasing the PurA concentration. A PurA concentration of $5.0 \times 10^{-4}$ was employed so that the ratio of the CL intensity to the blank $C L$ intensity $(S / B$ ratio) was maximum.

The influence of the $\mathrm{H}_{2} \mathrm{O}_{2}$ concentration on the $\mathrm{CL}$ intensity was examined over the $\mathrm{H}_{2} \mathrm{O}_{2}$ concentration range from $5.0 \times 10^{-3} \mathrm{M}$ to $5.0 \times 10^{-2} \mathrm{M}$. The $\mathrm{CL}$ intensity and the blank $C L$ intensity were increased along with an increase in the $\mathrm{H}_{2} \mathrm{O}_{2}$ concentration. At $3.0 \times 10^{-2} \mathrm{M}$ $\mathrm{H}_{2} \mathrm{O}_{2}$, the $S / B$ ratio was maximum. The optimum $\mathrm{H}_{2} \mathrm{O}_{2}$ concentration was $3.0 \times 10^{-2} \mathrm{M}$. HRP gave similar results to those of ARP.

Next, calibration curves for HRP and ARP were prepared under the optimized conditions. The results are shown in Fig. 4. The $\log -\log$ plots of the CL intensity against the amount of POD in the cuvette were linear over the ranges from a detection limit of $10 \mathrm{fmol}$ to $5.0 \mathrm{pmol}$ for ARP, and $20 \mathrm{fmol}$ to $5.0 \mathrm{pmol}$ for HRP, with the same slope of 0.77 . The detection limit was defined as the amount of POD, yielding a CL intensity equal to twice the blank CL intensity. The relative 
Table 2 Detection limit of POD with several CL reagent

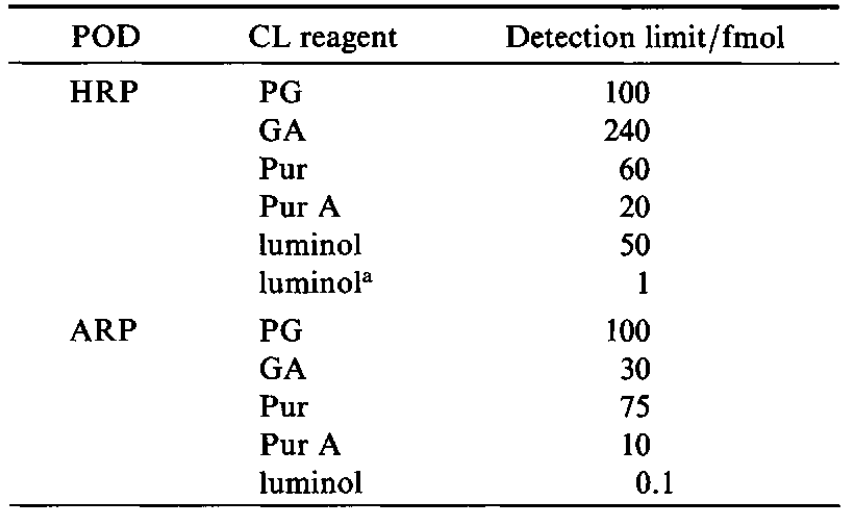

a. $p$-Iodophenol was used as an enhancer.

standard deviation of the five successive determinations was $3.1 \%$ and $3.3 \%$ at a level of $0.30 \mathrm{pmol}$ ARP and HRP, respectively.

Table 2 lists the detection limits of POD with polyphenolic $\mathrm{CL}$ reagents and luminol. In Table 2 , the CL conditions of each $C L$ reagent are optimized as well as that of PurA. Apparently, PurA was the most sensitive polyphenolic CL reagent for POD. In the detection limit of HRP, PurA was comparable to luminol without the use of an enhancer. The use of an enhancer in luminol CL markedly lowered the detection limit. On the other hand, luminol was much superior to PurA for ARP detection. In the CL reaction of polyphenol compounds, the CL intensities were almost independent on the origin of POD, except for GA.

\section{Effect of albumin on CL intensity}

In order to confirm the possibility of using PurA for clinical analysis, the CL reaction of PurA was performed by using a $0.30 \mathrm{pmol}$ HRP solution in the presence of $0.016 \%(\mathrm{w} / \mathrm{v})$ of bovine serum albumin (BSA). The CL response curves along with luminol are shown in Fig. 5. BSA had no significant effect on the CL response of PurA (Fig. 5(a)). On the other hand, BSA markedly increased the blank CL of luminol. This fact indicates that the luminol-CL is interfered by BSA. PurA would be superior to luminol in the CL detection of POD or its conjugates in the presence of proteins.

In conclusion, we have proposed a strategy for the design of a polyphenolic CL reagent for POD. The introduction of a carboxyl group decreased the blank CL intensity. Since PurA CL was less subject to an inhibition of BSA, the PurA method would be more practical for the detection of POD and its conjugates.

\section{References}

1. E. Ishikawa, T. Kawai and K. Miyai (eds.), "Kouso Meneki Sokuteihou (Enzyme Immunoassay, in Japanese)", 3rd ed., Igaku-shoin, Tokyo, 1987.
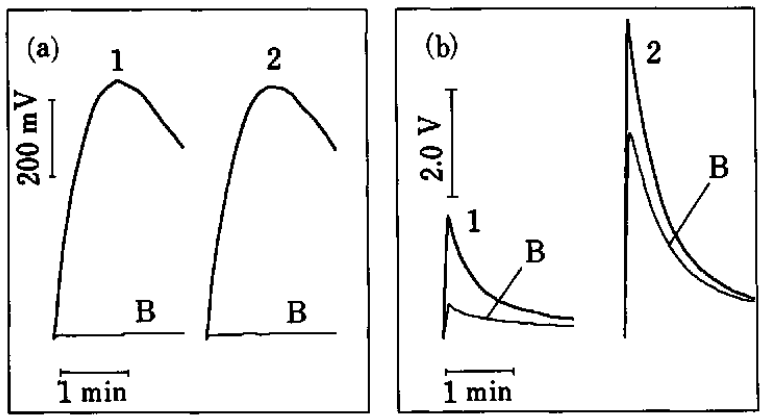

Fig. 5 Effect of bovine serum albumin on the CL response of PurA and luminol. (a) PurA, $[\operatorname{PurA}]=5.0 \times 10^{-4} \mathrm{M}$; $\left[\mathrm{H}_{2} \mathrm{O}_{2}\right]=3.0 \times 10^{-2} \mathrm{M} ;$ pH $7.0, \quad \mathrm{HRP}=0.30$ pmol. (b) luminol, [luminol] $=2.0 \times 10^{-5} \mathrm{M} ;\left[\mathrm{H}_{2} \mathrm{O}_{2}\right]=7.5 \times 10^{-4} \mathrm{M} ; \mathrm{pH}$ $9.0, \mathrm{HRP}=0.30 \mathrm{pmol}$. Curve 1 , control; curve 2 , in the presence of $0.016 \mathrm{wt} \%$ bovine serum albumin; $B$ represents a blank CL response.

2. E. Ishikawa, "Kouso Hyousiki-hou (Enzyme Labeling Method, in Japanese)", Gakkai Shuppan Center, Tokyo, 1991.

3. M. Renz and C. Kurz, Nucl. Acid Res., 13, 745 (1985).

4. H. Arakawa, M. Maeda and A. Tsuji, Anal. Biochem., 97, 248 (1979).

5. H. Arakawa, M. Maeda and A. Tsuji, Steroids, 38, 453 (1981).

6. R. Nilsson, Acta Chem. Scand., 18, 389 (1964).

7. M. Halmann, B. Velan and T. Sery, Appl. Environ. Microbiol., 34, 473 (1977).

8. B. Velan and M. Halmann, Immunochemistry, 15, 331 (1978).

9. M. Halmann, B. Velan, T. Sery and H. Schupper, Photochem. Photobiol., 30, 165 (1979).

10. G. H. G. Thrope and L. J. Kricka, Methods Enzymol., 133, 331 (1986).

11. T. P. Whitehead, G. H. G. Thorpe, T. J. N. Carter, C. Groucutt and L. J. Kricka, Nature [London], 305, 158 (1983).

12. A. Tsuji and T. Kanno (eds.), "Kagakuhakkou Imunoassei (Chemiluminescent Immunoassay, in Japanese)", Life Science, Tokyo, 1992.

13. R. A. W. Stoff and L. J. Kricka, in "Bioluminnescence and Chemiluminescence", ed. J. Scholmerich, R. Andereesen, A. Kapp, M. Ernst and W. G. Woods, p. 237, John Wiley, Chichester, 1987.

14. W. D. Crow, R. D. Harworth, J. Chem. Soc., 1951, 1325.

15. B. B. Kim, V. V. Apisarev and A. M. Egolov, Anal. Biochem., 199, 1 (1991).

16. D. Slawinska and J. Slawinski, Anal. Chem., 47, 2101 (1975).

17. N. P. Evmiridis, Analyst [London], 112, 825 (1987).

18. T. Matsuura, "Sanso Sanka Han-nou (Oxidation Reaction by Oxygen, in Japanese)", p. 181, Maruzen, Tokyo, 1977.

19. D. Dobos, "Electrochemical Data", pp. 310 and 317, Elsevier, New York, 1975. 